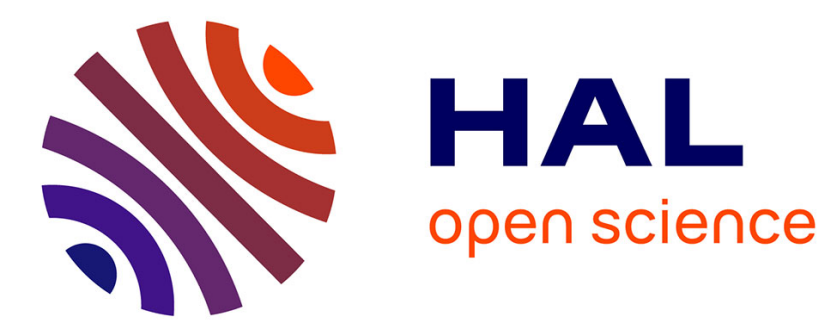

\title{
Une intégration maîtrisée des fonctions environnementales dans l'aménagement de la forêt domaniale du Romersberg.
}

Robin Degron, Christophe Gallemant

\section{- To cite this version:}

Robin Degron, Christophe Gallemant. Une intégration maîtrisée des fonctions environnementales dans l'aménagement de la forêt domaniale du Romersberg.. Revue forestière française, 1999, 51 (sp), pp.138-148. 10.4267/2042/5491 . hal-03443501

\section{HAL Id: hal-03443501 https://hal.science/hal-03443501}

Submitted on 23 Nov 2021

HAL is a multi-disciplinary open access archive for the deposit and dissemination of scientific research documents, whether they are published or not. The documents may come from teaching and research institutions in France or abroad, or from public or private research centers.
L'archive ouverte pluridisciplinaire HAL, est destinée au dépôt et à la diffusion de documents scientifiques de niveau recherche, publiés ou non, émanant des établissements d'enseignement et de recherche français ou étrangers, des laboratoires publics ou privés. 


\title{
UNE INTÉGRATION MAITTRISÉE DES FONCTIONS ENVIRONNEMENTALES DANS L'AMÉNAGEMENT DE LA FORÊT DOMANIALE DU ROMERSBERG
}

\author{
R. DEGRON - C. GALLEMANT
}

\section{PRÉAMBULE}

Cet article est dédié à la mémoire de Jean-Marie Liégeois, forestier de terrain, responsable de la forêt du Romersberg, qui nous a brutalement quittés à la fin de l'année 1998.

Son excellente connaissance du massif, et de son avifaune, son ouverture d'esprit et sa disponibilité ont permis une réalisation optimale des études auxquelles il a été associé, soit comme participant, soit comme guide de ceux qui les ont menées. Sa forte implication dans les choix de gestion du nouvel aménagement, puis dans leur mise en œuvre concrète en forêt, a également été déterminante pour l'exemplarité de cette opération-pilote.

La poursuite de celle-ci et la valorisation de ses résultats sont, pour nous tous, le meilleur témoignage de reconnaissance que nous puissions lui apporter.

Si la gestion des forêts publiques françaises respecte traditionnellement le principe de multifonctionnalité et de gestion durable, l'émergence depuis le début des années 1970 de la sensibilité environnementale des Français, relayée depuis peu sur le plan international, implique une réflexion forestière renouvelée conduisant éventuellement à une évolution maîtrisée des techniques d'aménagement et des pratiques sylvicoles. Fortement médiatisés, mondialisés, les débats sur l'accroissement de l'effet de serre, la destruction des forêts tropicales, la notion de développement durable rendent propices les amalgames, les incompréhensions. Grâce à l'aménagement forestier, le gestionnaire doit intégrer les nouvelles influences, élargir sa réflexion et définir une gestion finalement améliorée, cohérente avec l'histoire forestière nationale, adaptée à la réalité du contexte local.

Le cas de la forêt domaniale du Romersberg, à proximité de l'étang de Lindre, dans la partie mosellane du Parc naturel régional de Lorraine, illustre les changements dans l'approche de l'aménagement forestier, en particulier dans la prise en compte de la biodiversité (figures 1 et 2, p. 139). Dans une région de haute tradition forestière, présentant un fort potentiel productif (Degron, 1998b), le 


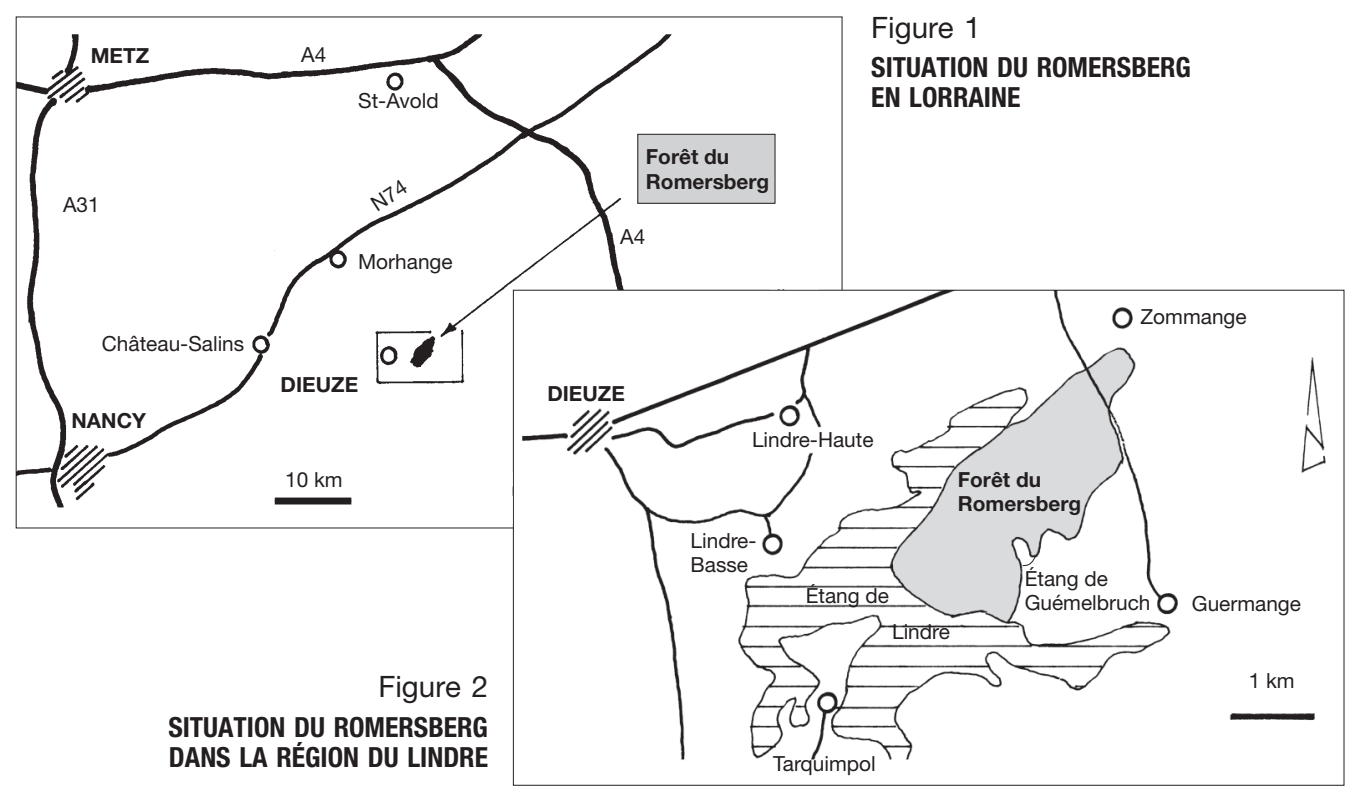

forestier doit savoir concilier la réalité des héritages, la permanence des contraintes économiques et le renouvellement d'une demande sociale exprimée notamment par les associations de protection de la nature.

Partant d'une situation d'affrontement au début des années 1990 entre sylviculteurs et naturalistes (ci-dessous), les acteurs régionaux et locaux trouvent un nouvel équilibre de gestion autour de l'aménagement révisé de 1996, dans la continuité d'une logique de production soutenue de bois (p. 141) et dans le respect de toute la richesse biologique du massif (p. 143).

\section{DU CONFLIT À LA DISCUSSION CONSTRUCTIVE : LA GENÈSE, LE CADRE ET LES ACTEURS D'UN AMÉNAGEMENT CONCERTÉ}

À partir d'un conflit entre sylviculteurs et protecteurs de l'avifaune cavernicole, une réflexion de fond est entamée sur les relations entre l'aménagement forestier et la biodiversité. Les débats bénéficient d'un cadre de concertation et de la réunion de nombreux spécialistes autour de l'aménagiste généraliste.

\section{Un conflit local entre forestiers et naturalistes}

Conformément à l'aménagement forestier du Romersberg révisé en 1990, une régénération est entamée dans la parcelle 13 du massif, visible depuis une petite route départementale : le peuplement de Chêne, issu d'un balivage intensif de taillis-sous-futaie, apparaît très enrichi et sensible à la dépréciation.

À proximité de l'étang de Lindre, réputé pour la qualité de son avifaune, la coupe d'ensemencement ne manque pas de faire réagir les naturalistes locaux qui voient là une atteinte à l'intégrité de l'écocomplexe lacustre et forestier. En référence aux problèmes pourtant très différents de déforestation tropicale, les indignations fusent; les banderoles s'étalent : " l'Amazonie, c'est aussi en France ». La forêt du Romersberg offre l'occasion d'une attaque en règle de la gestion forestière 
publique, relayée par la presse régionale. Le Gobe-mouche à collier (Ficedula albicollis) (photo p. 141), petit passereau forestier, oiseau cavernicole inféodé aux chênaies d'Europe centrale, rare en France (1), devient le symbole de l'opposition écologiste qui voyait à l'époque, en forêt du Romersberg, un site exceptionnel pour l'espèce.

\section{La mise en place d'un cadre de concertation}

Passée la première réaction de surprise, les forestiers locaux de l'Office national des Forêts (ONF) appuyés par la Direction départementale de Moselle, puis la Direction régionale de Lorraine, recherchent à nouer les fils du dialogue. Sans rentrer dans une vaine polémique avec les naturalistes, ils mettent en place le cadre d'une concertation, préalable indispensable à une réflexion constructive, source de progrès et d'enrichissement de leurs pratiques mais aussi d'explication de leurs contraintes auprès du public écologiste souvent mal informé des réalités économiques forestières.

Le Parc naturel régional de Lorraine, avec lequel l'ONF vient de signer une convention de coopération, apparaît comme une précieuse enceinte de médiation. Le Professeur Jean-Claude Rameau, responsable de la chaire d'écologie forestière de I'ENGREF Nancy, apporte son patronage à la réflexion naissante sur le massif et offre ses compétences scientifiques : il s'impose comme l'arbitre des intérêts forestiers et naturalistes. Réunis autour de sa présidence, un comité de pilotage se constitue finalement : ONF, PNRL, Association de protection de la nature, en l'occurrence Conservatoire des Sites lorrains (CSL) et naturalistes indépendants peuvent sereinement travailler ensemble.

\section{Une riche réflexion scientifique et technique préalable à la révision d'aménagement de la forêt domaniale}

Conscients des insuffisances de leur analyse du milieu naturel, les forestiers profitent, grâce aux compétences réunies au sein du comité de pilotage, d'apports scientifiques de grande qualité. Une étude pluridisciplinaire est lancée afin de connaître précisément les différents éléments constituant le patrimoine naturel forestier et d'évaluer l'impact de la gestion passée et future sur la biodiversité.

L'approche écologique retenue, ainsi que la perspective d'une redéfinition ou du moins d'une amélioration de l'aménagement forestier, mobilisent l'ensemble des acteurs. De 1991 à 1996, le travail réalisé est considérable ; les échanges sont intenses : 2 à 3 réunions annuelles sont organisées.

Les analyses forestières classiques sont particulièrement poussées : description au quart d'hectare des peuplements, définition d'une typologie des stations spécialement adaptée au massif qui puisse servir de référence aux études naturalistes, inventaire des milieux forestiers humides et associés (peuplement à ormes lisses du bord d'étang, zones humides intraforestières ou mardelles), approche de la diversité floristique des lisières.

De nombreuses études naturalistes offrent par ailleurs une large vue sur la richesse des écosystèmes forestiers : inventaire ornithologique sur l'ensemble des 420 ha du massif (avec une attention particulière pour l'avifaune cavernicole), études des peuplements d'insectes (Coléoptères, Lépidoptères), analyse des différentes populations de carnivores, de chiroptères, d'amphibiens.

En 1995, une synthèse des différents regards portés sur la forêt est réalisée : face à la multitude de données et à la grande richesse des analyses, le forestier prend le temps de structurer la connaissance engrangée dans la perspective de la révision d'aménagement.

(1) Des études ultérieures menées conjointement par la LPO (Ligue pour la protection des oiseaux) et l'ONF ont montré que, si la Lorraine est bien la limite occidentale de l'aire de répartition du gobe-mouche à collier, il n'y est pas rare et qu'il est présent dans presque toutes les chênaies du Plateau lorrain, ainsi que, plus à l'ouest, dans certaines forêts de la Woëvre. 
En marge des études stationnelles, sylvicoles et naturalistes, l'histoire de la forêt domaniale du Romersberg est étudiée afin de replacer sur le temps long les évolutions du massif et d'enrichir l'interprétation de certains résultats naturalistes concernant l'entomofaune saproxylophage.

En parallèle à la réflexion d'aménagement, une analyse économique est par ailleurs diligentée afin d'estimer l'impact des scénarios de gestion possibles.

Au-delà de l'intérêt technique de telles approches, l'impact financier des études mérite évidemment d'être discuté : les coûts engendrés sont à souligner et permettent de relativiser les efforts consentis exceptionnellement sur la forêt du Romersberg.

Ainsi en 1996, ce sont environ 1 million de francs qui auront été investis, y compris le coût des personnels de l'ONF. Ramené aux 420 ha du massif, $2400 \mathrm{~F} / \mathrm{ha}$ auront finalement été dépensés, soit quatre à cinq fois le coût moyen de révision des aménagements dans les forêts domaniales de Lorraine.

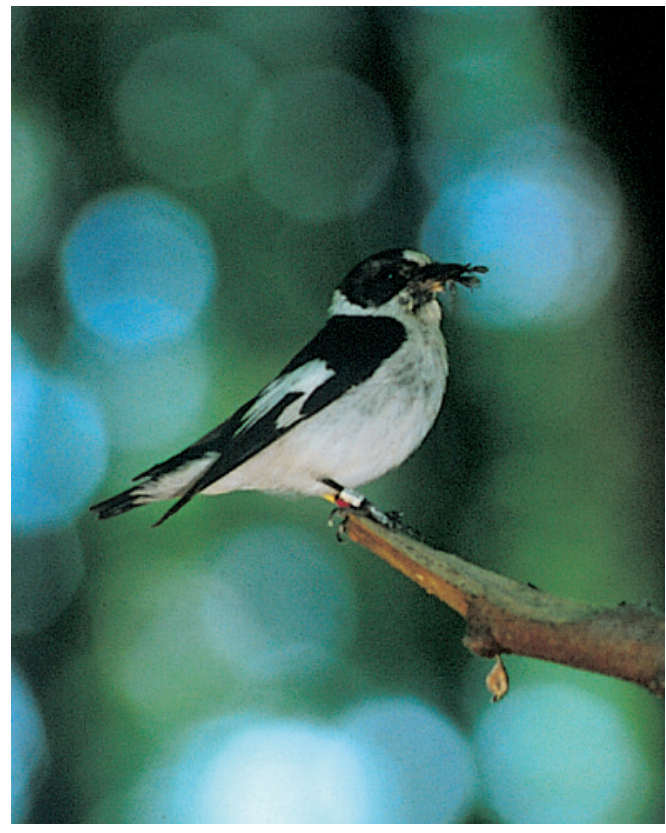

Le Gobe-mouche à collier Photo Y. MULLER

\section{UNE RÉVISION D’AMÉNAGEMENT EN CONTINUITÉ DES PLANS DE GESTION PRÉCÉDENTS}

Définie dans le cadre de la politique forestière nationale et dans le prolongement des efforts entrepris par les aménagistes et les sylviculteurs des périodes antérieures, la révision d'aménagement s'appuie, pour un massif donné, sur l'analyse du milieu naturel et du contexte socio-économique local ; elle réoriente ou conforte les objectifs de la gestion forestière et programme un plan d'action à moyen terme économiquement viable, à suivre tout au long de la période d'aménagement classiquement fixée à 15 ans dans les forêts domaniales de Lorraine.

Profitant d'un très grand nombre d'expertises naturalistes et sylvicoles, le plan de gestion de la forêt du Romersberg défini pour la période 1996-2010 permet de conforter la ligne directrice jusquelà suivie dans l'application de la méthode d'aménagement en futaie régulière appliquée à des peuplements à dominante Chêne issus ou en cours de conversion (2).

\section{La continuité historique et l'intérêt pratique de la méthode d'aménagement en futaie régulière en forêt domaniale du Romersberg}

Bien que les sources d'archives fassent défaut sur les origines de la conversion en futaie régulière de la forêt du Romersberg du fait des annexions allemandes de la Moselle de 1871 et 1940, il semble que le passage du régime de taillis à celui de futaie se soit ici préparé tout au long du $\mathrm{XIX}^{\mathrm{e}}$ siècle par enrichissement progressif de la réserve et que les premières coupes de régénération

(2) L'aménagement de la forêt domaniale du Romersberg (1996-2010) peut être consulté au Centre national de Documentation forestière, ENGREF-Nancy. II comprend notamment une synthèse et la liste exhaustive des différentes études réalisées sur le massif. 


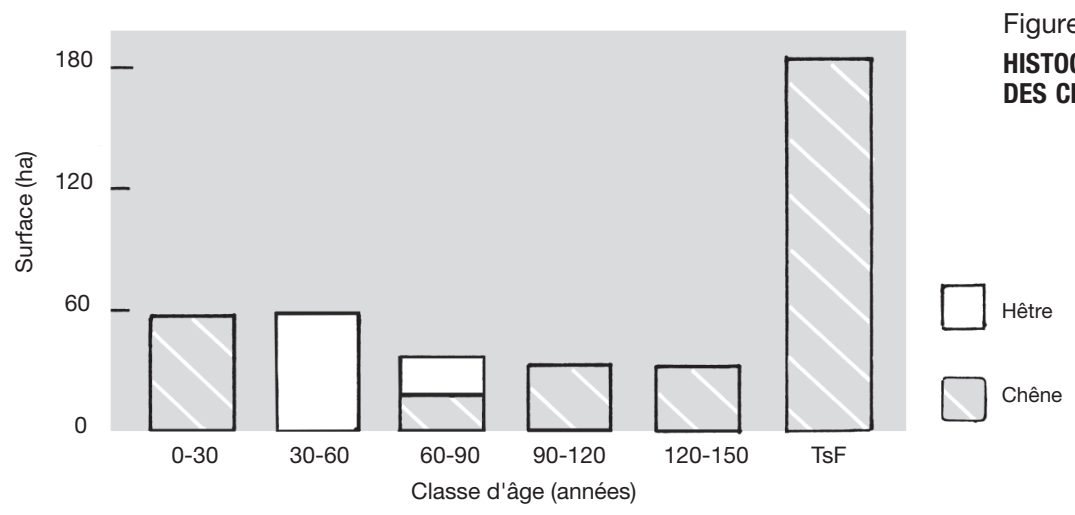

remontent à la fin du Second Empire (Degron, 1999). Depuis lors, les forestiers prussiens et français ont gardé le cap et maintenu l'objectif à très long terme d'une futaie régulière de chênes et de hêtres. En 1995, sur les 420 ha du massif, environ 200 ha de taillis-sous-futaie très enrichis restent à convertir (le capital sur pied moyen des peuplements issus du taillis-sous-futaie est compris entre 250 et $300 \mathrm{~m}^{3} /$ ha selon les parcelles) ; le solde des peuplements s'inscrit dans un cycle de futaie du stade semis à moyenne futaie (Gaudin, 1992) (figure 3, ci-dessus).

Relativement simple à mettre en œuvre, la méthode d'aménagement en futaie régulière permet d'obtenir grâce aux glandées partielles, dont la fréquence est d'environ six ans sur le Plateau lorrain, une régénération suffisante de Chêne mélangée pour l'essentiel de Hêtre et de Frêne. Bien adaptée à la rationalisation des travaux, la gestion pratiquée dans le massif répond au double impératif de renouvellement de la ressource et de maîtrise des coûts.

\section{Les intérêts écologiques de la méthode d'aménagement en futaie régulière en forêt domaniale du Romersberg}

Les études naturalistes commanditées en préalable à la révision d'aménagement tentent de décrire les relations entre la gestion sylvicole et la biodiversité forestière.

Que ce soit sur le plan floristique, entomologique, ornithologique ou mammologique, les différentes analyses écologiques mettent en évidence une relation positive entre diversité des peuplements et diversité biologique : à chaque stade de développement de la futaie correspond par exemple un cortège avien spécifique. Les semis ou les fourrés sont autant de milieux ouverts ou buissonnants favorables par exemple au Pipit des arbres ou au Bruant jaune ; les plus vieux peuplements de futaie seulement âgés de 120 à 150 ans commencent à accueillir des oiseaux cavernicoles (Gobemouche à collier, Pic mar). Les anciens taillis-sous-futaie, forcément riches en vieux arbres, sont évidemment particulièrement intéressants pour l'avifaune cavernicole (Morhain, 1991 ; Muller, 1993).

L'essence dominante Chêne sessile, naturellement favorisée par l'exploitation passée en taillis et par la conversion de grandes unités de gestion, apparaît comme une source de diversité biologique remarquable, particulièrement attractive pour les insectes corticaux (Meyer et al., 1994). Moins hégémonique que le Hêtre, le Chêne laisse la porte ouverte à un large mélange d'essences que ce soit sur le plan des feuillus nobles (Hêtres, Frênes, Érables, Merisiers) que des espèces secondaires (Tilleuls, Trembles, Charmes). Bien que le Chêne ne soit pas l'essence climacique du Plateau lorrain, sa prédominance, produit de l'histoire sylvicole, induit une biodiversité forestière maximale qui serait sans doute remise en cause si la dynamique naturelle pouvait se développer au profit du Hêtre. Les notions de biodiversité et de naturalité méritent ici d'être bien distinguées : en concerta- 
tion avec les membres du comité de pilotage, c'est l'objectif de maintien, voire d'amélioration de la diversité biologique, qui est prioritairement assigné à la révision d'aménagement du massif.

Finalement, dans le respect des héritages, par souci de simplicité de gestion et aussi compte tenu de la biodiversité induite par la conversion des peuplements issus de taillis-sous-futaie à dominante Chêne, l'aménagement forestier de la période 1996-2010 maintient le cap vers la constitution d'une futaie régulière de chênes relativement mélangée grâce aux techniques de régénération naturelle. Dans la perspective d'une production de bois soutenue, basée sur une exploitation progressive et extensive de la ressource, le terme de la conversion de l'ensemble du massif est fixé à 90 ans : plus que ce qui était prévu lors du précédent plan de gestion où la durée de renouvellement n'était que de 50 ans.

En marge de ces lignes directrices de la gestion forestière, des amendements sont apportés au système classique de la futaie régulière afin de perfectionner la prise en compte de la biodiversité dans la gestion sylvicole.

\section{LES AMENDEMENTS DE GESTION APPORTÉS AU PROFIT DE LA FONCTION ÉCOLOGIQUE FORESTIÈRE}

$\mathrm{Si}$, dans la mesure où elle est bien conduite, de façon équilibrée dans le temps, la conversion des taillis-sous-futaie en futaie régulière peut répondre à l'objectif fondamental de gestion durable de l'écomatériau bois, force est de reconnaître qu'elle gêne le développement de certaines composantes de l'écosystème forestier, en particulier les espèces inféodées aux très vieux peuplements et aux bois en décomposition. De par la relative rapidité du cycle de production du Chêne sessile fixé en Lorraine à 180 ans et du fait de la purge périodique des arbres dépérissants, certains facteurs d'enrichissement de la biodiversité sont absents de nos massifs. En forêt du Romersberg, le phénomène est d'autant plus marqué que les exploitations outrancières réalisées à la fin du $\mathrm{XVIII}$ siècle au profit des salines royales de Dieuze ont quasiment éliminé la possibilité d'avoir aujourd'hui des arbres bicentenaires et au-delà (Degron, 1995).

Dans la mesure où l'essence dominante Chêne est adaptée aux contraintes stationnelles du massif, profitant de sols argilo-limoneux profonds, et par conséquent qu'elle est peu sensible aux problèmes phytosanitaires relevant souvent d'agents biologiques opportunistes, le choix a été fait de développer la composante de très vieux peuplements et de bois dépérissants dans le cadre de la révision d'aménagement.

\section{Le maintien de vieux arbres dans les parcelles classées en régénération}

Étant donné que la forêt du Romersberg accueille de nombreux gobe-mouches à collier et pics mar, oiseaux reconnus pour leur intérêt patrimonial au niveau européen (cf. Directive Oiseaux de 1979), l'objectif fixé par le comité de pilotage à l'aménagement est de maintenir la population au niveau de référence, arbitrairement choisi, des années 1990.

À cette fin, des sur-réserves à vocation strictement écologique sont conservées dans les parcelles du groupe de régénération de la période d'aménagement. À terme, au fur et à mesure des révisions successives, toutes les unités de gestion du massif seront théoriquement concernées. La densité des sur-réserves est fixée à 2 arbres/ha : elle correspond à la densité totale des oiseaux cavernicoles calculée dans les parcelles les plus âgées de la forêt. Au sein d'une parcelle, les arbres réservés sont choisis de façon à ce qu'ils soient répartis uniformément : le Gobe-mouche à collier est particulièrement sensible au problème de compétition territoriale avec les autres oiseaux. En théorie, les sur-réserves sont repérées dès la première coupe secondaire. Le choix final reste ainsi 
ouvert jusqu'à la coupe définitive : les marteleurs peuvent adapter leur désignation à l'évolution de l'exploitation et de la perception qu'ils ont des arbres constituant le peuplement. Les chênes troués, au houppier équilibré, susceptibles de tenir au vent et économiquement sans grande valeur sont privilégiés lors de cette désignation d'arbre "sans avenir". Dans les deux premières parcelles où ont été sélectionnées des sur-réserves écologiques, la sélection s'est faite tardivement : il s'agissait d'une opération de rattrapage. Compte tenu de l'état d'avancement des exploitations, toute la surface des parcelles concernées n'a pu être couverte par les deux réserves à l'hectare.

Il est encore trop tôt pour tirer des enseignements de cette technique de génie écologique : le traitement en rattrapage et le faible échantillonnage des parcelles traitées limitent évidemment la portée des premières observations réalisées en dehors d'un cadre scientifique rigoureux. Les résultats sur le maintien des populations cavernicoles sont d'ailleurs contrastés selon la parcelle considérée. Au terme de la révision d'aménagement, un nouvel inventaire avifaunistique devrait être réalisé sur le modèle de celui qui a été effectué au début des années 1990 et a établi l'état initial des populations du massif (Muller, 1993 op. cit.).

Le coût d'opportunité représenté par la non-récolte des sur-réserves de Chêne est évalué à environ $20 \mathrm{~F} / \mathrm{ha} / \mathrm{an}$ qui sont à rapporter aux $1200 \mathrm{~F} / \mathrm{ha} / \mathrm{an}$ de bénéfice brut prévisionnel (hors frais de gestion) de la gestion forestière sur 15 ans (1996-2010).

En marge de l'intérêt pour l'avifaune d'un tel dispositif, on peut remarquer que les sur-réserves ainsi sélectionnées permettront à terme d'atteindre la densité de 2 arbres morts/ha, référence affichée par le guide sur la prise en compte de la biodiversité édité par l'Office national des Forêts en 1994. Les cavernicoles et les espèces inféodés à la nécromasse devraient a priori bénéficier de cette mesure de gestion forestière intégrée.

\section{L'implantation d'îlots de vieillissement dans le massif}

Étant donné la faiblesse des populations d'insectes saproxylophages au sein de la forêt du Romersberg, conséquence de l'histoire moderne du massif et des coupes sanitaires pratiquées depuis le $\mathrm{XIX}$ siècle, l'objectif de retrouver une composante de très vieux peuplements, riches en nécromasse, potentiellement favorables au retour des espèces rares qui y sont liées, est affiché par le comité de pilotage de l'aménagement. Cétonidés, Lucanidés et Cérambycidés sont les familles d'insectes visées par l'objectif d'enrichissement de la biodiversité forestière.

Pour atteindre ce résultat, des îlots de vieillissement sont implantés sur environ $10 \%$ de la surface du groupe de régénération : l'îlot prend concrètement la forme d'un petit bouquet compact d'un demi à un hectare (figure 4, p. 145). Au fur et à mesure des révisions successives d'aménagement, $10 \%$ du massif devraient a priori être sélectionnés. Le seuil de référence est défini arbitrairement : c'est le fruit de la négociation entre les forestiers et les naturalistes. Le terme du maintien des îlots de vieillissement est par ailleurs fixé au double de l'âge d'exploitabilité de l'essence objectif : 360 ans pour des peuplements à dominante Chêne sessile. En fait, il s'agit par le biais de cette mesure de rallonger sur une partie de la forêt la durée du cycle de production des chênes et d'intégrer, au sein d'un massif essentiellement de production, un maillage de peuplements très âgés : « imiter la Nature, sans partout hâter son œuvre » telle pourrait être la devise de cette révision d'aménagement.

Le suivi des îlots de vieillissement et de leur influence sur l'entomofaune n'est évidemment pas facile à envisager, ne serait-ce qu'à cause du temps nécessaire à une telle évaluation et de la difficulté scientifique de sa réalisation. C'est avant tout un pari qui est tenté en ayant conscience d'œuvrer de toute manière en faveur d'une restauration partielle et maîtrisée de la biodiversité forestière. Le débat sur la conduite des îlots de vieillissement jusqu'à leur terme arbitraire est très ouvert. Si aucune coupe n'intervient dans les peuplements, les hêtres déjà présents en sous-étage 


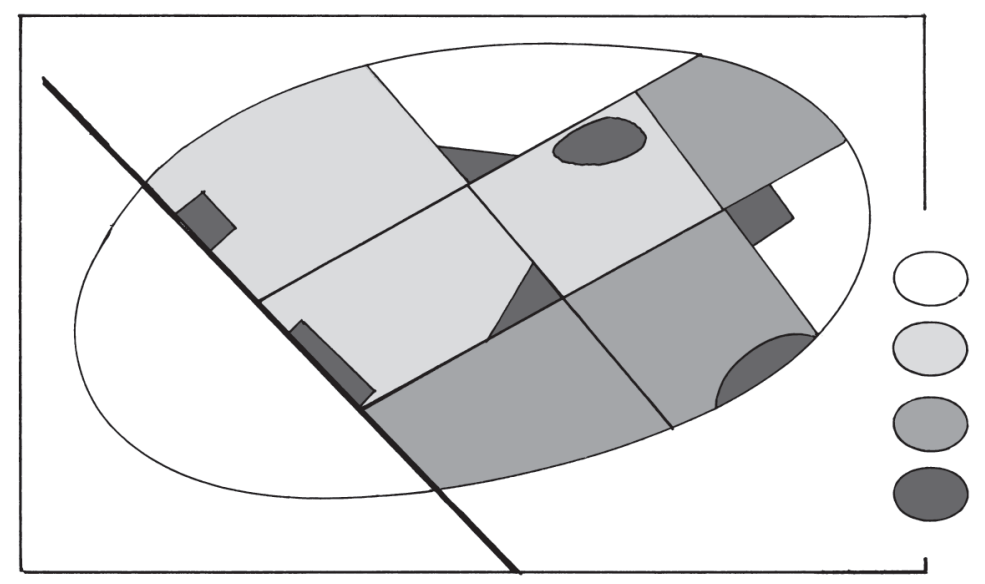

Un maillage de très vieux peuplements au sein d'une forêt de production

Jeunes peuplements

Moyens peuplements

Vieux peuplements

Îlots de vieillissement

Histogramme théorique des peuplements d'une chênaie comprenant 10 \% d'îlots de vieillissement

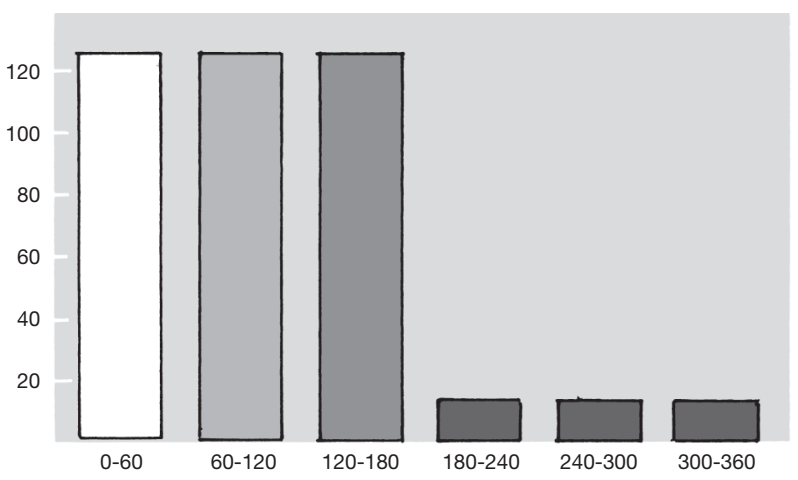

prendront tôt ou tard le dessus sur les chênes et l'objectif de maintien de la prédominance du Chêne compte tenu de son intérêt biologique ne pourra être atteint : la non-intervention n'est donc pas ici une solution de gestion pertinente. On peut envisager également d'améliorer les îlots au sens traditionnel du terme en essayant d'éduquer une très vieille futaie, riche en gros arbres pas forcément dépérissants : dans ce cas, des exportations de biomasse de Chêne sont à prévoir. Cette alternative est défendue par Jean Dubourdieu dans le manuel d'aménagement de l'Office national des Forêts de 1997. Elle s'oppose peu ou prou à l'objectif de production de nécromasse et par là au redéveloppement partiel d'une composante structurante d'une chênaie écologiquement normale. En accord avec le comité de pilotage, le choix fait dans le cadre de l'aménagement-pilote, tout à fait expérimental, de la forêt domaniale du Romersberg est de prévoir une intervention minimum contre les hêtres de sous-étage susceptibles de dominer le peuplement de l'îlot ; aucune coupe de Chêne n'a été prévue.

Le coût d'opportunité représenté par la non-récolte des îlots de vieillissement est évalué à environ $100 \mathrm{~F} / \mathrm{ha} / \mathrm{an}$. Avec la non-recette correspondant au maintien des sur-réserves, le "coût environnemental spécifique" de l'aménagement du Romersberg représente $10 \%$ du bénéfice net (hors frais de gestion) de la gestion forestière sur 15 ans (1996-2010).

En marge de l'intérêt écologique des îlots de vieillissement, on peut aussi concevoir par ce biais la possibilité de préserver l'environnement d'un arbre remarquable ou de maintenir très longtemps une perspective paysagère jugée intéressante. En forêt du Romersberg, certains îlots ont été implantés 
en bordure de l'étang de Lindre afin de maintenir un écrin forestier continu en périphérie de ce site reconnu remarquable par l'Atlas régional des paysages de Lorraine établi par la Direction régionale de l'Environnement en 1995.

\section{CONCLUSIONS}

Même si elle n'est pas généralisable à l'ensemble des forêts publiques, l'expérience conduite en forêt du Romersberg se poursuit. Elle offre d'ores et déjà de nombreux enseignements et participe à la formation des aménagistes, sylviculteurs, naturalistes, acteurs d'une gestion forestière de progrès. Elle conforte par ailleurs l'intérêt de la démarche d'aménagement forestier comme outil irremplaçable de développement durable.

Le suivi de l'aménagement et des paris techniques tentés se poursuit dans le cadre du comité de pilotage qui continue de se réunir chaque année. Au-delà des observations périodiques et ponctuelles concernant les populations d'oiseaux cavernicoles, les chauves-souris, les carnivores, les amphibiens et les insectes, une étude générale du massif est envisagée lors de sa prochaine révision d'aménagement prévue en 2010 afin de mesurer l'impact global des choix techniques arrêtés. Réalisée en 1997, une description arbre par arbre des îlots de vieillissement complète l'état "zéro" du massif.

S'il apparaît difficile de généraliser la démarche entreprise en forêt du Romersberg et les résultats scientifiques obtenus, compte tenu des caractéristiques propres du massif et des coûts économiques engendrés, en particulier sur le plan des études, certains enseignements semblent cependant pouvoir enrichir la réflexion forestière globale ; ils confortent certaines recommandations techniques générales dans les forêts publiques.

Le constat le plus important est que, bien au-delà du débat, souvent stérile, sur les effets respectifs des différents modes de traitement sur la diversité biologique, le principal impact de la gestion forestière sur la biodiversité concerne la raréfaction des biocénoses liées aux vieux arbres et au bois mort. Cela n'a rien d'étonnant puisqu'un des principes chers aux forestiers, français notamment, est " d'imiter la nature et de hâter son œuvre ". En hâtant l'œuvre de la nature, c'est-à-dire en récoltant les arbres avant que leur bois ne se décompose, il est évident que le forestier raccourcit le cycle naturel de la forêt et agit négativement sur tous les êtres vivants inféodés au stade "d'écroulement" des peuplements forestiers.

Les mesures préconisées pour maintenir et, si possible, restaurer la diversité biologique liée au bois mort, aux vieux arbres et aux vieux peuplements sont d'ailleurs reprises à l'ONF, soit dans des instructions ou guides de gestion, soit dans la dernière version du manuel d'aménagement : maintien d'arbres morts dans l'ensemble des peuplements, choix et maintien de sur-réserves dans les parcelles en régénération, implantations d'îlots de vieillissement. Les deux premières mesures ont un impact économique très limité ; leur généralisation à la plupart des forêts publiques, voire privées peut donc s'envisager sans difficulté. Par contre, le coût d'opportunité que représentent les îlots de vieillissement n'est pas négligeable (environ 10 \% du revenu brut pour le cas de la forêt du Romersberg).

Trop souvent ignoré par les forestiers comme par les naturalistes, le poids de l'Histoire dans l'explication des peuplements et de la biodiversité mérite par ailleurs d'être souligné. Cette conclusion, qui rejoint celle d'autres études récentes, tels les travaux d'Étienne Dambrine de l'INRA Champenoux, doit inciter à s'intéresser de plus près à cette science humaine.

Enfin, le travail considérable réalisé pour la révision d'aménagement du massif représente un précieux investissement en terme de communication et de formation. Depuis 1996, l'expérience du 
Romersberg fait l'objet de publications, de communications, en France comme à l'étranger, de sessions de formation destinées tant aux forestiers de l'ONF qu'à ceux de la forêt privée et aux naturalistes.

Des forestiers et scientifiques de plus de 20 nationalités différentes sont venus visiter la forêt. Et, depuis 1998, une information destinée au grand public sur l'aménagement-pilote de la forêt du Romersberg est disponible sous forme d'une plaquette et sur le site internet de l'ONF. Un document de synthèse, très complet, sur l'ensemble des études et travaux menés est en cours de préparation.

En élargissant la réflexion sur la place de l'aménagement au sein de la politique forestière française, l'intérêt de l'outil fondamental de gestion durable nous semble conforter par l'expérience du Romersberg. D'une part, car les grandes orientations sylvicoles prises depuis plus d'un siècle et assurées par la ligne des aménagements successifs ont résisté à l'épreuve de la discussion avec les naturalistes, aujourd'hui mieux informés de la logique forestière ; d'autre part, car la démarche d'aménagement a su faire la preuve de sa capacité à intégrer de nouveaux enjeux par la voie du compromis et de la synthèse de regards variés. Garants des équilibres forestiers sur le temps long, à l'écoute de la demande sociale, les forestiers contribuent, par le dialogue, à l'intégration maîtrisée des nouvelles fonctions environnementales au sein d'un objectif de développement durable du patrimoine national.
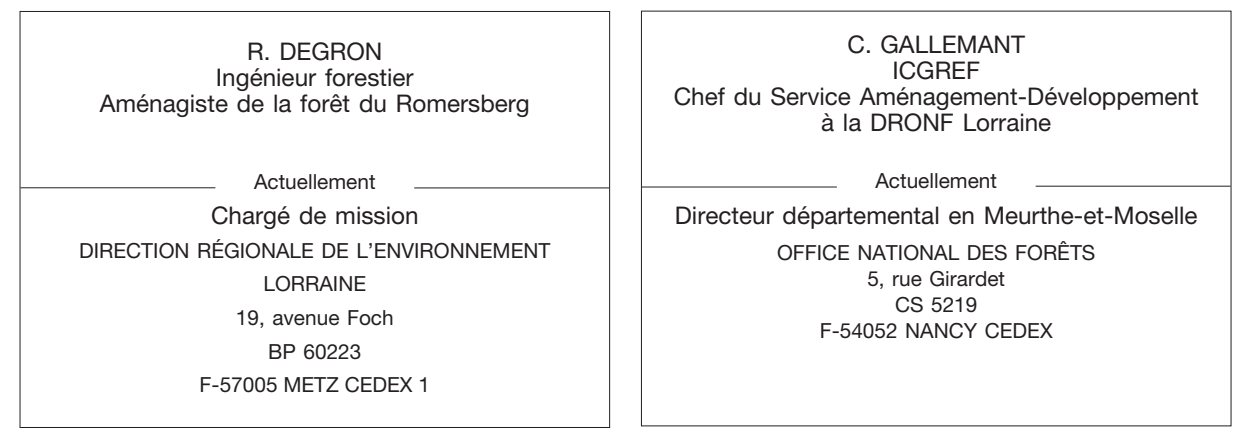

\section{BIBLIOGRAPHIE}

DEGRON (R.). - Les Conversions forestières de Lorraine : bilan, contrastes, rythmes et ruptures. - Nancy : Université de Nancy II, 1999. - 500 p. (Thèse de Doctorat en Géographie).

DEGRON (R.). - L'Héritage sylvicole allemand dans le Plateau lorrain mosellan. - Saint-Julien-les-Metz : Archives départementales de la Moselle, 1998b. - La Forêt de Moselle - Feuilles d'Archives, pp. 73-81.

DEGRON (R.). - Historique de la forêt du Romersberg : une forêt de Lorraine sous l'emprise des salines. Revue forestière française, vol. XLVII, $n^{\circ}$ 5, 1995, pp. 590-597.

DUBOURDIEU (J.). - Manuel d'aménagement forestier. - Paris : Office national des Forêts ; Lavoisier, 1997. $244 \mathrm{p}$.

GAUDIN (S.). - Contribution à une étude écologique forestière globale : typologie des peuplements de la forêt domaniale du Romersberg (Moselle). - Nogent-sur-Vernisson : ENITEF, 1992. - 89 p. + annexes (Mémoire de $3^{\mathrm{e}}$ année). 
MEYER (M.), BRAUNET (C.), DOLISY (D.), DURGMAND (D.). - Étude des patrimoines naturels forestiers de la forêt domaniale du Romersberg (Moselle). Partie entomologique. Inventaires qualitatifs et quantitatifs des bioindicateurs de milieux forestiers parmi les Coléoptères : Carabidae, Cerambycidae, Lucanidae, Staphylinidae. Rapport intermédiaire. - Pont-à-Mousson : Parc naturel régional de Lorraine, 1994. - $21 \mathrm{p}$.

MORHAIN (E.). - Forêt domaniale du Romersberg (Moselle). Contribution à l'étude de la valeur biologique et écosystémique. Proposition de gestion. - Dijon : ENITA, 1991. - 62 p. + annexes (Mémoire de $3^{e}$ année).

MULLER (Y.). - Expertise avifaunistique de la forêt du Romersberg. - Fénétrange : Conservatoire des Sites lorrains, 1993. - 40 p. + annexes.

OFFICE NATIONAL DES FORÊTS. - Guide pour la prise en compte de la diversité biologique dans l'aménagement et la gestion forestière. - Paris : Office national des Forêts, 1993. - $32 \mathrm{p}$.

\section{UNE INTÉGRATION MAÎTRISÉE DES FONCTIONS ENVIRONNEMENTALES DANS L’AMÉNAGEMENT DE LA FORÊT DOMANIALE DU ROMERSBERG (Résumé)}

La forêt domaniale du Romersberg est une chênaie-hêtraie de 420 ha située à proximité de l'étang de Lindre (Moselle) gérée, jusqu'en 1990, selon un aménagement forestier de production classique.

Partant d'une situation d'affrontement entre sylviculteurs et naturalistes liée à la mise en régénération d'une parcelle, les acteurs régionaux et locaux trouvent un nouvel équilibre de gestion autour d'une révision d'aménagement concertée, réalisée en 1996. Celle-ci assure la continuité d'une logique de production soutenue de bois tout en intégrant la richesse biologique du massif.

Bien qu'expérimentales, non généralisables à l'ensemble des forêts publiques, les techniques d'aménagement et de sylviculture employées alimentent la réflexion du forestier et enrichissent concrètement sa palette d'intervention.

\section{THE CONTROLLED INTEGRATION OF ENVIRONMENTAL USES IN THE MANAGEMENT OF THE STATE FOREST OF ROMERSBERG (Abstract)}

The state forest of Romesberg comprises 420 ha of oak and beech stands located in the vicinity of the Lindre pond (Moselle) which until 1990 was managed according to a conventional production system.

Starting from of a situation of conflict between foresters and naturalists pertaining to a regeneration plan for a plot, the regional and local players were able to achieve a balanced management approach through a co-operatively revised development plan in 1996. It allows for continuity with the sustained timber production rationale while building on the biological assets of the massif.

Although the planning and sylvicultural techniques used are experimental and cannot be generally applied to all public forests, they do provide foresters with food for thought and broaden their range of practical interventions. 